\title{
On characterisation of finitary algebraic categories
}

\section{Francis Borceux and B.J. Day}

\begin{abstract}
The aim of this article is to characterise categories which are $V$-algebraic (equals $V$-theoretical) over $V$ where $V$ is a symmetric monoidal closed category satisfying suitable limitcolimit commutativity conditions (basicly axiom $\pi$ ).
\end{abstract}

\section{Introduction}

In the theory of finitary $V$-algebraic categories over a category $V$ satisfying axiom $\pi$ (Borceux and Day [3]) there are two basic characterisation theorems. The first of these is discussed in Borceux and Day [4], Section 2.5, and is based on the concept of rank of a functor.

The aim of this paper is to describe the second characterisation theorem which is closer to the original characterisation theorem of Lawvere for $V=$ Ens (see Diers [7], Corollary 5.5.6). This second theorem is based on the notion of a suitable strong projective generator in the category; namely the free algebra on $I \in V$ when the category is known to be algebraic.

In Section 3 we develop the theory of near-cartesian closed categories. The principal example of such a category is the category of pointed $k$-spaces; the tensor product in this category is the "smash product" $X \# Y$ of pointed spaces $X$ and $Y$ and while this is not the cartesian product there are canonical diagonals $X \rightarrow X \# X \# X \# \ldots \# X$. This allows us to deduce, from the characterisation theorem, that all operadic categories on pointed $k$-spaces are algebraic (that is,

Received 11 November 1977. 
theoretical). The main interest here derives from the well-known fact that in the theory of infinite loop spaces it is possible to use theories (Boardman and Vogt [2] and Beck [1]) or operads (May [11] and Kelly [9]). We also point out other instances where this phenomenon occurs.

Throughout the article we assume that $V=(V, \otimes, I,[-,-], \ldots)$ is a complete and cocomplete symmetric monoidal closed category satisfying axiom $\pi$, and we assume that all categorical algebra is relative to $V$ unless otherwise stated. We assume some familiarity with Borceux and Day [3] and [4]. The basic algebra appears in [6] and [7].

\section{Preliminaries}

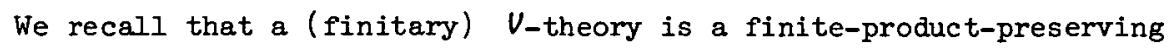
functor $t: v_{f}^{\text {op }}+T$ which is one-one on objects, where $v_{f}$ denotes the full subcategory of $V$ comprising the finite copowers of $I \in V$. Each $V$-theory $(T, t)$ generates a monad $T=T(T)$ on $V$ which has the property

$$
\int^{V_{f}}[n, X] \otimes[m, T n] \cong[m, T X]
$$

for all $m \in V_{f}$, and is thus the "restriction to $v$ " of the monadic adjunction $t^{*} \rightarrow\left[t^{o p}, 1\right]:[T, v]+\left[v_{f}^{o p}, v\right]:$

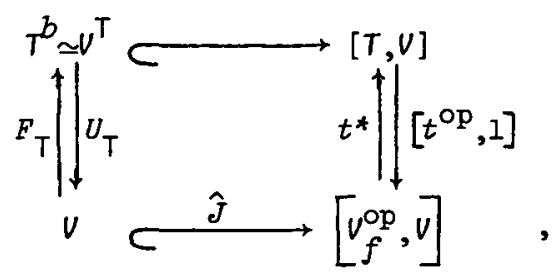

where $J: V_{f} \rightarrow V$ is the canonical inclusion. We say that $T(T)$ has 8lgebraic rank $J$. By Day [5], Theorem 2.1, and the density of $J$, it follows that $V^{\top}$ is category equivalent to the full subcategory $T^{b}$ comprising the finite-product-preserving functors from $T$ to $V$.

PROPOSITION 1.1. Let $t: V_{f} \rightarrow T$ be $a$-theory and let $A$ be $a$ small category with finite products. Let $G: A \rightarrow V$ be a finite-product 
preserving functor and let $H: A^{\mathrm{OP}}+T^{b}$ be any functor. Then the mean tensor product $G A * H A$ exists in $T^{b}$ and is isomorphic to $\int^{A} C A \otimes H A$ in $[T, V]$.

Proof. Iterated use of axiom $\pi$ gives us

$$
\begin{aligned}
{\left[m, \int^{A} G A \otimes H A(t)\right] } & \cong \int^{A} G A \otimes H A(t I)^{m} \\
& \cong \int^{A} G A \otimes H A(t m),
\end{aligned}
$$

as required for $\int^{A} G A \otimes H A$ in $[T, V]$ to in fact be a $T$-algebra. //

We also recall from Borceux and Day [4] that if $(T, t)$ is a

commutative $V$-theory then $T$ has a canonical symmetric monoidal structure $\otimes: T \otimes T \rightarrow T$ such that $t: V_{f}^{o p} \rightarrow T$ preserves tensor products.

PROPOSITION 1.2. If $(T, t)$ is a commutative $U$-theory then $T^{b}$ is a symmetric monoidal closed category enriched over $U$.

Proof. Clearly $T^{b}$ is closed under exponentiation in $[T, V]$, because the internal-hom is given by $[A, B]=\int_{T}\left[A\left(t_{n}\right), B(\operatorname{tn} \otimes-)\right]$ which preserves finite products whenever $B$ is a $T$-algebra. The unit object is the free $T$-algebra on $I \in V$, namely $T(t l,-)$. The tensor product of two algebras $A$ and $B$ is given by

$$
\begin{aligned}
A \otimes B & =\int^{T \otimes T} A\left(t_{m}\right) \otimes B(t n) \otimes T(t m \otimes t n,-) \\
& \cong \int^{T} A(t m) \otimes \int^{T} B(t n) \otimes T(t m \otimes t n,-) .
\end{aligned}
$$

But, for each fixed $m, \int^{T} B\left(t_{n}\right) \otimes T(t m \otimes t n,-)$ is a $T$-algebra; so let it be $B(t m)$ in Proposition 1.1. This then shows that $A \bar{\otimes} B$ is again a T-algebra. Thus the convolution structure on $[T, V]$ restricts to $T^{b} . / /$

This result was established in Borceux and Day [4] but is recalled here for convenience in section 3 . 


\section{Structure-semantics and characterisation}

We denote by $A d g=A d g(J)$ the category whose objects are functors $U: B \rightarrow V$ having a left $J$-adjoint and whose morphisms are functors $M: B \rightarrow B^{\prime}$ such that $U^{\prime} M=U$ (see Diers [6], Section 4). The functor $\left(J_{-}\right)$semantics

$$
\text { Sem }: T h^{\text {op }} \rightarrow A d g
$$

is given by $\operatorname{Sem}(T)=\left(v^{\top}, U_{T}\right)$.

THEOREM 2.1. Semantics Sem : $T h^{\circ p} \rightarrow$ Adg is fully faithful and has a Zeft adjoint.

Proof. This is just the V-analogue of Diers [6], Theorem 4.2.

The left adjoint is the structure functor

$$
\text { Str : Adg } \rightarrow T h^{O P}
$$

which maps $F \underset{J}{-} U: B \rightarrow V$ to the obvious algebraic theory generated by $F \frac{J}{J} U$. We have

$$
\begin{aligned}
& \varepsilon: \operatorname{Str} \text { Sem } \cong 1: T h^{\text {op }} \rightarrow T h^{o p}, \\
& n: 1 \Rightarrow \text { Sem Str : Adg } \rightarrow \text { Adg } .
\end{aligned}
$$

THEOREM 2.2. Given $F \vec{J} U: B \rightarrow V$, then $B$ is algebraic with respect to $U$ if $B$ is cocomplete, $U$ reflects isomorphisms, and $U$ preserves $G A$ * $H A$ whenever $A$ is a small category with finite products, $G: A+V$ is a finite-product-preserving functor, and $H: A^{\mathrm{OP}} \rightarrow B$ is a functor.

Proof. Note first that, using the fact that Str (Sem Str) $\cong S t r$, we obtain a functor $H: T^{\mathrm{OP}} \rightarrow B$ such that

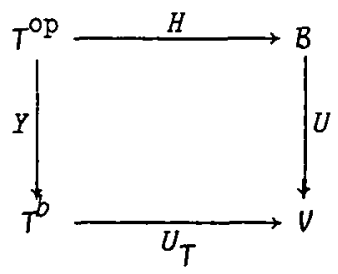


commutes, and such that $\operatorname{Lan}_{Y} H$ is left adjoint to $n_{B}: B \rightarrow T^{b}$. Thus, since $B$ is cocomplete, $\eta_{B}$ has a left adjoint $\sigma$ which is the restriction to algebras of a left adjoint $\bar{\sigma}$ to $B \rightarrow[T, V]$; namely $\bar{\sigma}(G)=\int^{T} G(t n) \cdot H(t n)$. We require

(i) on $\cong I: B \rightarrow B$, and

(ii) $1 \cong n \sigma: T^{b} \rightarrow T^{b}$.

Because $U$ reflects isomorphisms, we require for (i) that $U \sigma n \cong U$. But $U_{T} \eta \cong U$, so we need $U_{\sigma} \cong U_{T}: T^{b} \rightarrow V ;$ this also guarantees (ii). Finally, to establish the result, consider

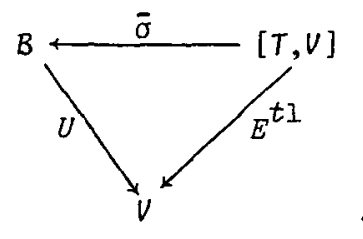

Then $\bar{\sigma}(G)=\int^{T} G(t n) . H(t n)$ for all $G \in[T, V]$. If $G \in T^{b}$, then

$$
\begin{aligned}
U\left\{\int^{T} G(t n) . H(t n)\right\} & \cong B\left(F 1, \int^{T} G(t n) . H(t n)\right\} \text { since } F \frac{J}{J} U, \\
& \cong \int^{T} G(t n) \otimes B\left(F 1, H\left(t_{n}\right)\right) \text { by hypothesis, } \\
& \cong \int^{T} G(t n) \otimes U H(t n) \\
& \cong \int^{T} G\left(t_{n}\right) \otimes T(T n, T 1) \text { by (*), } \\
& \cong G(t 1) \text { by the representation theorem, } \\
& =U_{T}(G) \text { as required. // }
\end{aligned}
$$

An object $P \in B$ is called an abstractly finite projective generator of $B$ if $B(P,-): B \rightarrow V$ reflects isomorphisms and preserves $G A * H A$ whenever $A$ is a small category with finite products, $G: A \rightarrow V$ is a finite-product-preserving functor, and $H: A^{O P}+B$ is any functor.

COROLLARY 2.3. Let $B$ be cocomplete with an abstractly finite 
projective generator $P$. Then $U=B(P,-): B \rightarrow V$ is algebraic.

Proof. The adjoint $F-\vec{J} U$ is given by $F(n)=n_{P}$, so the result follows from the theorem.

COROLLARY 2.4. Let $V$ be a r-category (see Borceux and Day [4], Definition 2.1.1). Then $B$ is algebraic over $V$ if and only if $B$ is cocomplete and has an abstractly finite projective generator.

Proof. Over a $\pi$-category any algebraic category is cocomplete, since it has coequalisers of reflective pairs. Moreover, FI is an abstractly finite projective generator of $T^{b}$ by Proposition $1.1 . \quad / /$

In conclusion we note that if $F \underset{J}{T} U: B+V$ and $U F: V_{f}+V$ has the structure of a monoidal functor then the theory of the structure of $U$ is commutative.

THEOREM 2.5, If $V$ is a $\pi$-categom, then $B$ is commutatively $V$-algebraic over $V$ if and only if $B$ is cocomplete cand has a symetric monoidal closed structure $(B, I, \otimes,[-,-], \ldots)$ whose identity object I is an abstractly finite projective generator of $B . \quad / 1$

\section{Example: near-cartesian closed categories}

The category of pointed compactly generated speces ( $k$-spaces) is more than just algebraic over compactly generated spaces. It is equipped with a canonical identification map $A \times B \rightarrow A \otimes B$ and this permits us to consider diagonals $A \rightarrow A \otimes \ldots \otimes A$. The key theoretical observation at this point is that if $T$ is a commutative $V$-theory over a closed category $V$ which satisfies axiom $\pi$ then, in the presence of a suitable diagonal functor $T \rightarrow T \otimes T$, the functor $\int^{T} A\left(t_{n}\right) \otimes T\left(t_{n} \otimes \ldots \otimes t n,-\right)$ is again a T-algebra and is, in fact, the $m$ th tensor power of $A$.

In order to formalise what we have in mind here, we introduce the following definition.

DEFINITION 3.1. The closed category $V$ is called near-cartesion if there exists an ordinary natural transformation $e_{A B}: A \times B \rightarrow A \otimes B$ such that the following diagrams commute: 
(1)

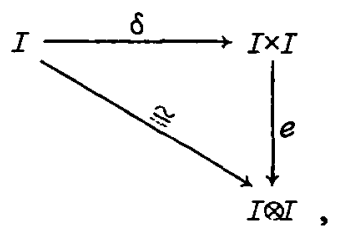

(2)

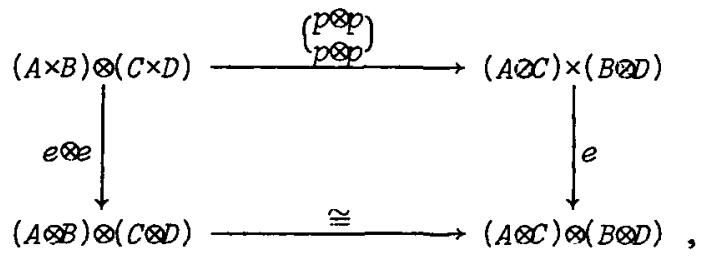

and

$$
\int^{n}[n, A] \otimes\left(\otimes^{m} n\right) \cong \otimes^{m} A \text { for all } m>0 \text { and } A \in V \text {. }
$$

Note that it is possible to write (3) because (1) and (2) imply the existence of a canonical functor $K=K(e): A \times B \rightarrow A \otimes B$ for any $V$-categories $A$ and $B$. The following consequence is easily established.

PROPOSITION 3.2. Let $V$ be near-cartesian and let $(T, t)$ be a commutative $v$-theory. Then the m-fold tensor power $(m>0)$ of a T-algebra $A$ is given by the formula

$$
A \otimes \ldots \otimes A=\int^{T} A\left(t_{n}\right) \otimes T\left(t_{n} \otimes \ldots \otimes t n,-\right)
$$

THEOREM 3.3. Let $(T, t)$ be a commutative theory over the nearcartesian closed category $V$ and suppose $V$ is a $\pi$-category. Let $R$ be a monad on $T^{b}$ generated by an operad on $T^{b}$. Then $\left(T^{b}\right)^{R}$ is algebraic over $V$.

Proof. For the concept of an operad we refer to May [11]. The important aspect here is that the endofunctor $R$ is given by an expression of the form $R A=\int^{n} S n \bar{\otimes}\left(\otimes^{n} A\right)$ where $n$ runs over either the free $V$-category on the integers or the free $V$-category on the permutation category (the integers are greater than or equal to 0 , with no morphisms $n \rightarrow m$ if $n \neq m$, and the morphisms $n \rightarrow n$ being the permutations on $n$ ). Let us denote the $V$-adjunctions involved by 


$$
V \underset{U}{\stackrel{F}{\rightleftarrows}} T^{b} \underset{U^{\prime}}{\stackrel{F^{\prime}}{\rightleftarrows}}\left(T^{b}\right)^{R}
$$

Both adjunctions $F \dashv U$ and $F^{\prime} \dashv U^{\prime}$ are monadic and both $U$ and $U^{\prime}$ create coequalisers of reflective pairs; hence $U U^{\prime}$ reflects isomorphisms and $\left(T^{b}\right)^{R}$ is cocomplete. Thus, by Theorem 2.2, it remains to check that $U U^{\prime}$ preserves $G A * H A$ whenever $A$ is a small category with finite products, $G: A \rightarrow V$ preserves finite products, and $H: A^{\circ p} \rightarrow\left(T^{b}\right)^{R}$ is any functor. But already $U$ preserves $G A * U^{\prime} H A$ so it remains to check that $R$ on $T^{b}$ preserves $G A * U^{\prime} H A$. For any $H^{\prime}: A^{\circ \mathrm{P}} \rightarrow T^{b}$ we have

$$
R\left(G A * H^{\prime} A\right)=\int^{n} S n \bar{\otimes}\left(\bar{\otimes}^{n}\left(G A * H^{\prime} A\right)\right),
$$

where

$$
\vec{\otimes}_{B}=\int^{T} B(t m) \otimes T(t m \otimes \ldots \otimes t m,-): T \rightarrow V .
$$

Thus

$$
\begin{aligned}
\vec{\otimes}^{n}\left(G A * H^{\prime} A\right) & \cong \int^{T}\left(\int^{A} G A \cdot H^{\prime} A\right)(t m) \otimes T(t m \otimes \ldots \otimes t m,-) \\
& \cong \int^{T}\left\{\int^{A} G A \cdot H^{\prime} A(t m)\right) \otimes T(t m \otimes \ldots \otimes t m,-) ;
\end{aligned}
$$

so

$$
\begin{aligned}
R\left(C A * H^{\prime} A\right) & \cong \int^{n} S n \otimes\left(\int^{A} G A \cdot\left\{\int^{T} H^{\prime} A(t m) \otimes T(t m \otimes \ldots \otimes t m,-)\right)\right\} \\
& \cong \int^{A} G A \cdot\left(\int^{n} S n \otimes \int^{T} H^{\prime} A(t m) \otimes T(t m \otimes \ldots \otimes t m,-)\right) \\
& \cong G A * R H^{\prime} A .
\end{aligned}
$$

Thus, by induction, we have $R^{p}\left(G A * H^{\prime} A\right) \cong G A * R^{p} H^{\prime} A$ for $p \geq 0$. Thus $U^{\prime}$ creates $G A * H A$, as required. //

In order to generate examples of near-cartesian closed categories we consider the following

DEFINITION 3.4. A symmetric monoidal monad $T=(T, \mu, n)$ on a cartesian closed category is called near-cartesian if the transformation 
$\tilde{T}: T X \times T Y \rightarrow T(X \times Y)$ is left inverse to the canonical transformation $\kappa: T(X \times Y) \rightarrow T X \times T Y$.

LEMMA 3.5. Let $V$ be cartesian closed and let $T=(T, \mu, \eta)$ be a near-cartesian monad on $V$. Suppose $T$ preserves coequalisers of reflective pairs and let $F+U$ denote the associated monoidal adjunction over $V$. Then $\tilde{U}_{A B}: U A \times U B \rightarrow U(A \otimes B)$ is a (regular epimorphic)

natural tronsformation in $V$.

We leave the proof to the reader as an exercise.

THEOREM 3.6. Let $V$ be cartesian closed and let $T=(T, \mu, \eta)$ be a finitary near-cartesian monad on $V$. Then $V^{\top}$ is a near-cartesian closed category.

Proof. $V^{\top}$ satisfies axiom $\pi$ by Borceux and Day [3]. To satisfy Definition 3.1 we choose $e_{A B}=\tilde{U}_{A B}$, using Lemma 3.5. Then, by Definition 3.1, (1) and (2) are simple consequences of applying $U$ and using the naturality of $\tilde{U}$. It remains to prove that

$$
\int^{F n}[F n, A] \otimes\left(\otimes^{m} F n\right) \cong \otimes^{m} A
$$

for all $m>0$ and $A \in V^{\top}$. By virtue of the diagram

$$
F^{2} A \times F^{2} A \stackrel{e}{\longrightarrow} F^{2} A \times F_{A}^{2} \rightarrow F A \otimes F \cong F(A \times A) \stackrel{\zeta}{\rightarrow} A \times A
$$

we have that $e$ is the coequaliser in $v^{\top}$ of a pair of morphisms $F^{2} A \times F^{2} A+A \times A$. We then have

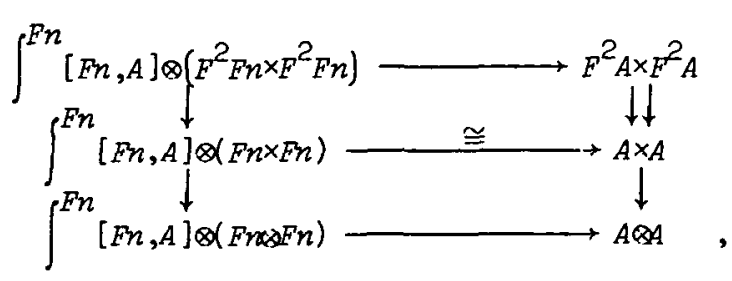


where the isomorphism follows from axiom $\pi$ on $v^{\top}$. For similar reasons the top moxphism is an epimorphism, so $\int^{E n}[F n, A] \otimes(F n \otimes F n) \cong A \otimes A$. The proof is analogous for $m>2$. /

EXAMPLE 3.7 ( $V$ cartesian closed). Let $A$ be a commutative semigroup in $V$ such that

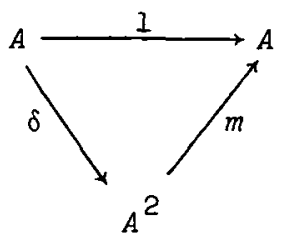

commutes (sometimes such an object is called a semilattice (without a unit)). Then $T X=X+A$ is a near-cartesian unary monad on $V$. Thus the category $A / V$ is near-cartesian closed.

EXAMPLE 3.8 ( $V$ cartesian closed). Let $G: V \rightarrow V$ be a symmetric monoidal finitary near-cartesian endofunctor on $V$ and let $\varepsilon: G \Rightarrow 1$ be a monoidal natural transformation. Then $T X=X+G X$ is near-cartesian and finitary. Thus the category "G/V" is near-cartesian closed.

\section{References}

[1] Jon Beck, "On H-spaces and infinite loop spaces", Category theory, homology theory and their applications III, 139-153 (Proc. Conf. Seattle Research Center, Battelle Memorial Institute, 1968, Volume Three. Lecture Notes in Mathematics, 99. Springer-Verlag, Berlin, Heidelberg, New York, 1969).

[2] J.M. Boardman and R.M. Vogt, "Homotopy-everything H-spaces", BuZl. Amer. Math. Soc. 74 (1968), 1117-1122.

[3] Francis Borceux and B.J. Day, "On product-preserving Kan extensions", Bul2. Austral. Math. Soc. 17 (1977), 247-255.

[4] Francis Borceux and Brian Day, "Universal algebra in a closed category", J. Pure Appl. Algebra (to appear). 
[5] B.J. Day, "Linear monads", BulZ. Austral. Math. Soc. 17 (1977), 177-192.

[6] Y. Diers, "Foncteur pleinement fidèle dense classant les algèbres" (Publications Internes de l'U.E.R. de Mathématiques Pures et Appliquées, 58. Université des Science et Techniques de lille I, 1975).

[7] Yves Diers, "Type de densité d'une sous-catégorie pleine", Arn. Soc. Sci. Bruxezzes Sér. I 90 (1976), 25-47.

[8] Samuel Eilenberg and G. Max Kelly, "Closed categories", Proc. Conf. Categorical Algebra, La Jolla, California, 1965, $421-562$ (Springer-Verlag, Berlin, Heidelberg, New York, 1966).

[9] G.M. Kelly, "On the operads of J.P. May", unpublished manuscript.

[10] S. Mac Lane, Categories for the working mathematicion (Graduate Texts in Mathematics, 5. Springer-Verlag, Berlin, Heidelberg, New York, 1971).

[11] J.P. May, The geometry of iterated Loop spaces (Lecture Notes in Mathematics, 271. Springer-Verlag, Berlin, Heidelberg, New York, 1972).

Institut de Mathématique pure et appliquée,

Université Catholique de Louvain,

Belgi um;

Department of Pure Mathematics,

University of Sydney,

Sydney,

New South Wales. 